\title{
Management of Pain and Related Disabilities in Primary Fibromyalgia Using Neuromodulator Techniques, Repetitive Transcranial Magnetic Stimulation and Transcranial Direct Current Stimulation
}

\author{
Mohja A. EL-Badawy*1, Mervat A. Reda ${ }^{2}$, Sahar Y. Ragab ${ }^{3}$, Dalia M Ezz-Eldin ${ }^{4}$ \\ Department of Physical Medicine, Rheumatology and Rehabilitation, \\ Ain Shams University Hospitals, Cairo, Egypt. \\ *Corresponding author: Mohja Ahmed Elbadawy, Mobile: (+20) 01005048716, Email:mohjaelbadawy@gmail.com
}

\begin{abstract}
Background: Fibromyalgia syndrome (FMS) is a chronic condition that causes pain, stiffness, and tenderness in muscles, tendons, and joints. It is also characterized by disturbed sleep, fatigue, anxiety, depression, and disturbances in bowel function. Management of FMS is at present very challenging as it contains multiple etiological factors and psychological tendencies; though, a patient-centered approach is essential to deal with this problem.

Objective: To compare the efficacy of repetitive transcranial magnetic stimulation (rTMS) and transcranial direct current stimulation (tDCS) in improving pain and related disabilities in patients with primary (1ry) FM.

Patients and Methods: Thirty patients with 1ry FM, 18 to 50 years old were randomized into 2 groups. Group I included 15 patients, who received 8 sessions (2/week for 4 consecutive weeks) of high frequency rTMS (10 Hz frequency) and Group II included 15 patients received 8 daily sessions of anodal tDCS ( $2 \mathrm{~mA}$ for 20 minutes) applied over the left primary motor cortex (M1).

Results: Pain visual analogue scale (VAS), the revised Fibromyalgia Impact Questionnaire (FIQR), tender point scale, and Hospital Anxiety and Depression Scale (HADS) showed a significant decrease between baseline and follow-up assessments for both groups. Moreover, a significant change in all assessment scales was observed posttreatment when compared between both groups with more significant improvement in the group that received rTMS. Conclusion: Both rTMS and anodal tDCS of the left primary motor cortex showed marked improvement in symptoms of pain, functional disabilities and psychological status in patients with 1ry fibromyalgia. Both neuromodulator techniques can be considered as promising alternatives therapeutic options in the management of pain and related disabilities in FM.
\end{abstract}

Keywords: Chronic pain, Fibromyalgia, Neuromodulator techniques, Repetitive transcranial magnetic stimulation, Transcranial direct current stimulation.

\section{INTRODUCTION}

Fibromyalgia (FM) is one of the most challenging chronic pain syndromes with regard to treatment, affecting $3 \%$ to $5 \%$ of the world's population ${ }^{(\mathbf{1}, 2)}$. It is characterized by widespread chronic musculoskeletal pain, accompanied by sleep disturbances, fatigue, decreased physical performance, and psychological distress that affects quality of life ${ }^{(3,4)}$. Many studies indicated that FM could be considered as one of the central sensitivity syndromes ${ }^{(5-7)}$.

Mhalla and colleagues, represented that FM is associated with impairment of intra cortical modulation, which supports the hypothesis that it may be associated with changes in cortical excitability ${ }^{(\mathbf{8})}$. Considering central pain-processing changes found in FM and the effects of neuromodulatory techniques on neuroplasticity, several studies had examined the effects of these techniques on chronic pain in FM ${ }^{\text {(9- }}$ 12).

Transcranial magnetic stimulation (TMS) is a non-invasive technique of brain stimulation that uses changes in magnetic fields to increase or decrease neuronal activity ${ }^{(13)}$. Low frequencies $(\leq 1 \mathrm{~Hz})$ can induce neuronal inhibitory function, whereas high frequencies $(\geq 5 \mathrm{~Hz})$ are associated with increased cortical excitability ${ }^{(\mathbf{1 3})}$. Repetitive stimulation to the left prefrontal cortex is frequently associated with antidepressant and mood stabilizer effects, while repeated stimulation of the primary motor cortex (M1), over contralateral side of the painful area, can produce analgesic effects through the activation of horizontal fibers in the superficial layers of the precentral gyrus ${ }^{(\mathbf{1 4}, 15)}$.

Another neuromodulator technique is the transcranial direct current stimulation (tDCS). Its work is based on weak electrical currents that are applied to the scalp through electrodes for a specific amount of time. Anodal stimulation produces membrane depolarization, and consequently increasing neuronal firing. While cathodal stimulation produces membrane hyperpolarization and consequently decreasing neuronal firing (16). The effectiveness of both neuromodulator techniques in managing pain and related functional and psychiatric impairments in FM has been reported in several trials (17-19). To the best of our knowledge, comparing the effects of both modalities in FM patients had not been previously studied. 
Therefore, the present study aims to compare the efficacy of repetitive transcranial magnetic stimulation (r-TMS), and transcranial direct current stimulation (t-DCS) as neuromodulator techniques in the management of pain, functional disabilities, and psychological impact in patients with primary (1ry) fibromyalgia (FM).

\section{PATIENTS AND METHODS \\ Ethical approval:}

The Ethical Committee of Authors Institution of Ain Shams University approved the present study, and all patients signed informed consent before participation.

This prospective randomized controlled clinical study included 30 patients diagnosed clinically with 1ry FM according to the American College of Rheumatology (ACR 2010) criteria ${ }^{(20)}$. All patients were recruited from the Outpatient Clinic of Physical Medicine and Rehabilitation Department in Authors Institution Hospitals. Inclusion criteria were as follows: 1ry FM patients aged between 18 and 50 years with VAS for pain $>5$.

Exclusion criteria included patients with a bad general condition, scalp skin pathology, major neurological illness as epilepsy, seizures, multiple sclerosis, parkinsonism or brain tumors, patients with pacemakers, metals in the scalp, eye or neck, metallic joint prosthesis, cochlear implants, and some artificial heart valves.

Pregnant or breast-feeding patients, patients with secondary (2ry) fibromyalgia, or those enrolled in another type of physical therapy program were also excluded.

The sample size was calculated on the initially collected 40 cases, with a confidence level of $90 \%$ and a $10 \%$ margin of error; the ideal sample was 26 cases (http://www.quattrics.com).

Randomization was done using the sealed envelope system. Accordingly, patients were randomized equally into 2 groups: Group I included 15 patients who underwent 8 sessions of rTMS applied over the left M1 area (2 sessions/week for 4 consecutive weeks). M1 was localized using the 10/20 International EEG system as $\mathrm{C} 3$ adapted by measuring $5 \mathrm{~cm}$ below the vertex and located along the coronal line over the left parietal cortex ${ }^{(\mathbf{2 1})}$ (Figure 1).

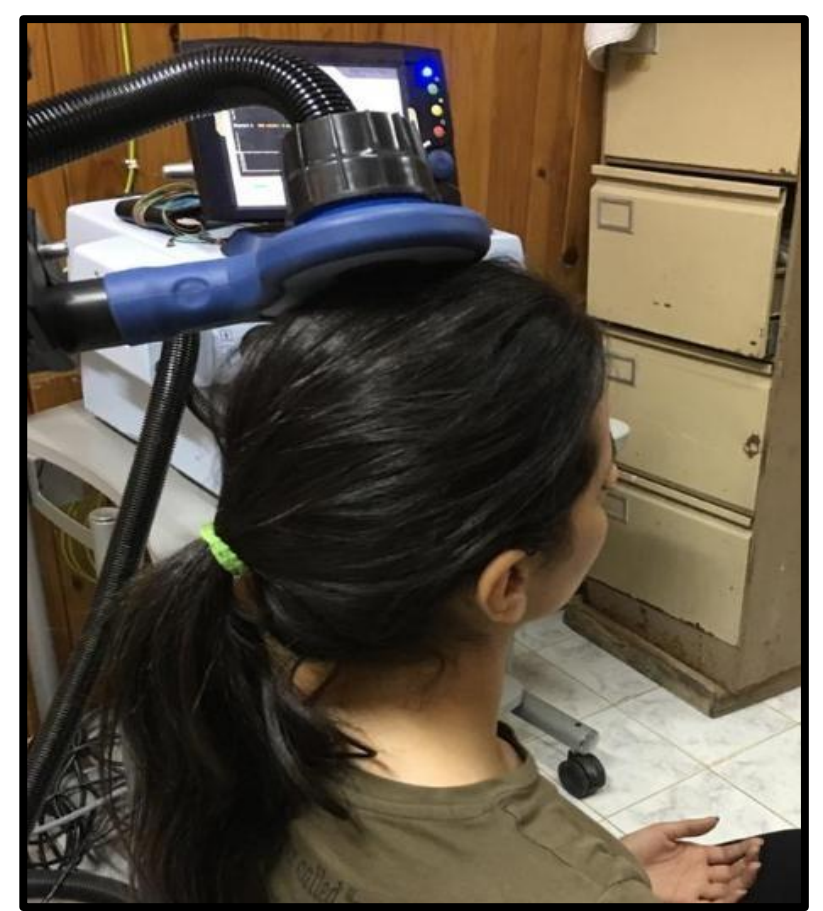

Figure (1): Localization of the transcranial magnetic stimulation coil over the left primary motor cortex.

A Magstim rapid II 8 shaped coil trans-cranial magnetic stimulator was used (Magstim Rapid Therapy system, Magstim Inc, USA). Each treatment session consisted of 25 series of 8 -second pulse trains, with 52 seconds intervals between series, at a stimulation frequency of $10 \mathrm{~Hz}$, two sessions per week for four consecutive weeks. Each subject's resting motor threshold (MT) was determined by using single TMS stimulation over the site of the motor cortex, with a positive response in the motor cortex determined by minimum power that induced movement of the contralateral thumb of the patient. To ensure subject comfort, maximal TMS power was set to be $90 \%$ of the motor threshold. But patients' comfort was assessed during the stimulation and if at any time the patient indicated significant discomfort from the stimulus, the power was decreased incrementally.

Group II included 15 patients who received 8 daily sessions of active anodal tDCS applied over the left M1 area with an intensity of $2 \mathrm{~mA}$, for 20 minutes in daily sessions (Activadose tDCS Device, ActivaTek, Inc, USA). The cathode is placed over the contralateral (right) supraorbital area. M1 is localized as C3 by using the 10/20 International EEG system. A pair of thick rectangular surface sponge electrodes were soaked in saline and applied to the scalp at the desired sites of stimulation (Figure 2). 


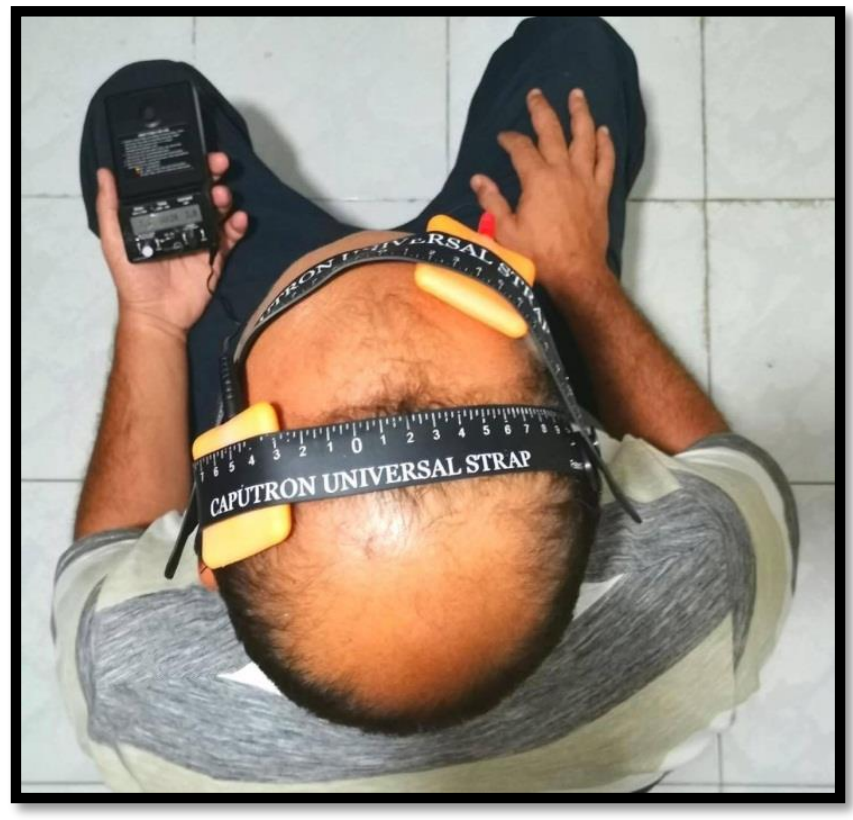

Figure (2): Electrodes placement for transcranial direct current stimulation showing the target location of the anodal electrode (C3; left) and the cathode electrode over the right supraorbital area

Minor side effects were reported from rTMS therapy including transient headache and tingling. The most common side effect of tDCS observed in our patients was a tingling sensation felt under the electrodes. Headache, and/or dizziness were also reported by some patients at the start of tDCS sessions but they were well tolerated. The primary outcomes assessed in this study were the pain visual analog scale (VAS) ${ }^{(22)}$ and the revised Fibromyalgia Impact Questionnaire (FIQR) ${ }^{(23)}$.

The FIQR is a self-administered evaluation instrument developed to measure FM patients' status, and progress. The questionnaire has 21 individual questions that are based on an 11-point numeric rating scale of 0 to 10 , with 10 being the 'worst'. All questions are designed to describe the past 7 days. It is composed of 3 domains. The first one consists of a physical functioning scale. Domain 2 determines the number of days that the patient feels well and the number of days they are not able to work because of FM symptoms. Domain 3 is a 10-centimeter VAS on which the patient rates the intensity of different fibromyalgia symptoms including memory affection, tenderness, balance abnormalities, and environmental sensitivity. The sum of scores in each domain is calculated. Then domain 1 is divided by 3 , domain 2 by 1 , and domain 3 by 2 . Finally, the three resulting domain scores are added to obtain the total FIQR ${ }^{(23)}$.
Secondary outcome measures included the tender point score ${ }^{(24)}$ and the Hospital Anxiety and Depression Scale (HADS) ${ }^{(25)}$. The tender point score was performed using digital palpation with an approximate force of $4 \mathrm{~kg}$. Pain elicited by digital palpation was found to be present in at least 11 out of 18 sites. For a tender point to be rated positive, the patient must state that the palpation was painful ${ }^{(24)}$. HADS consists of two subscales that are scored separately, one for measuring anxiety and the other for measuring depression. Each subscale is composed of seven items. Each item was rated by the patient on a 4-point ( $0-3)$ scale, so the possible scores ranged from 0 to 21 for each of the two subscales. The HADS manual indicates that a score (0-7) is "normal," (8-10) is "mild," (11-14) is "moderate," and (15-21) is "severe" (25).

\section{Statistical analysis}

Statistical analysis including data collection, revision, coding, and entering into the Statistical Package for the Social Sciences (IBM SPSS version 20, Armonk, NY: IBM Corp). The qualitative data were shown as numbers and percentages whereas quantitative data were presented as mean, standard deviations, median, and interquartile range (IQR). The comparison between the two groups with qualitative data was done by using the Chi-square test.

The comparison between two independent groups with quantitative data and parametric distribution was performed by using an independent ttest. Paired $t$-test was a test of significance used for comparison between paired data normally distributed having nominal variables. Mann-Whitney U Test was a test of significance used for comparison between two groups having quantitative variables without normal distribution (for non-parametric data). P-value was considered significant as the following: $\mathrm{P}<0.05$ : Significant, $\mathrm{P}<0.01$ : Highly significant and $\mathrm{P}<$ 0.001: Very highly significant.

\section{RESULTS}

This study was conducted on 30 patients divided equally into two groups. The flow chart of patients is demonstrated in Figure (3). We found no significant difference in demographic data, wide spread pain index (WPI), symptom severity (SS) scale between both patients' groups. The pre-treatment VAS, Tender point score, FIQR, and, HADS anxiety and depression subscales were statistically comparable in both groups (Table 1). 


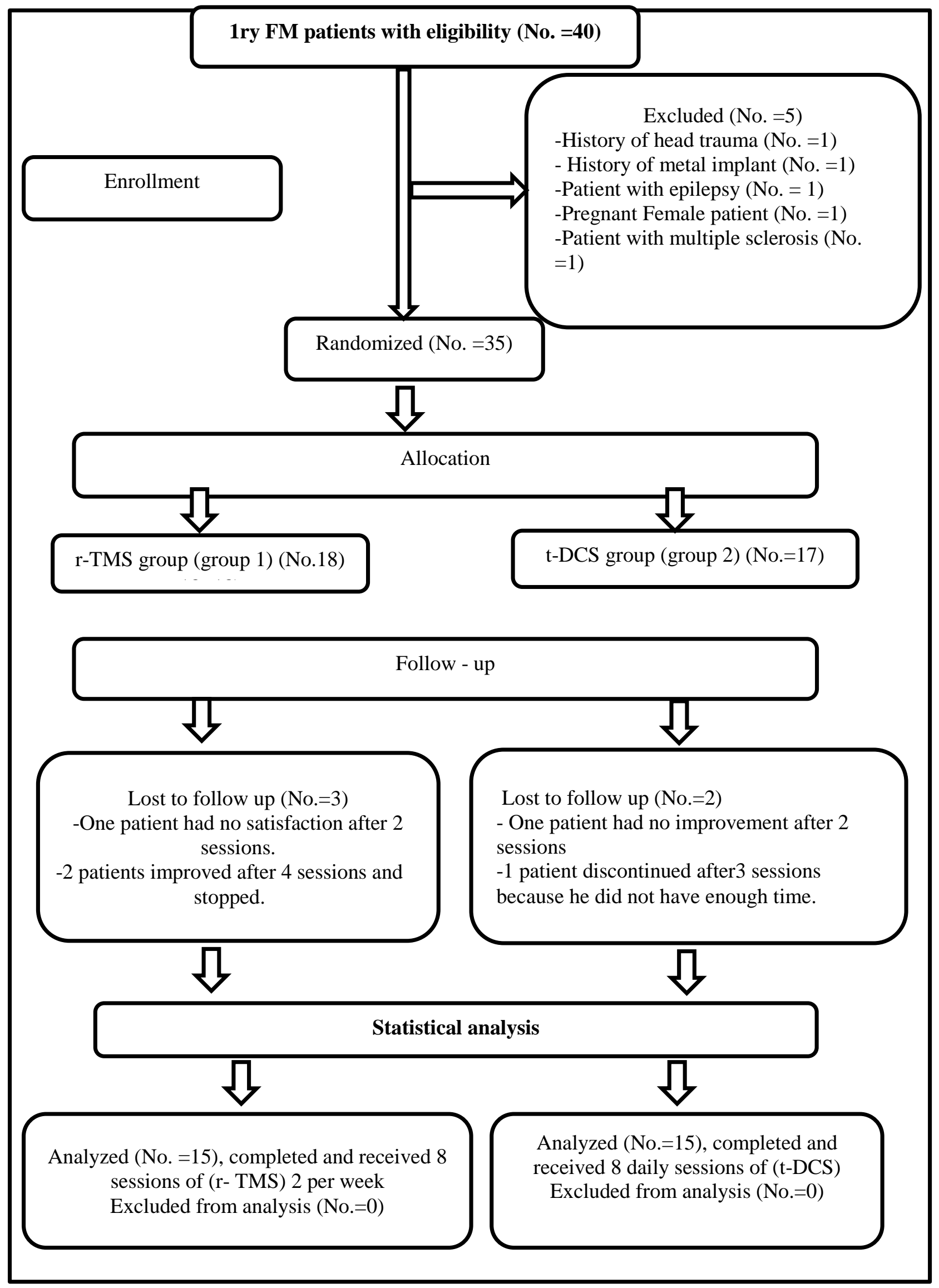

Figure (3): Flow chart of patients. 
Table (1): Comparison of demographic data, WPI, SS scale and all clinical scales between both patients' groups

\begin{tabular}{|c|c|c|c|c|c|c|}
\hline \multirow{2}{*}{\multicolumn{2}{|c|}{ Demographic and Clinical data }} & \multicolumn{2}{|c|}{$\begin{array}{c}\text { Group I } \\
\text { r-TMS therapy } \\
(\mathrm{N}=15)\end{array}$} & \multicolumn{2}{|c|}{$\begin{array}{c}\text { Group II } \\
\text { t-DCS therapy } \\
(N=15)\end{array}$} & \multirow[t]{2}{*}{$P$ - value } \\
\hline & & Mean & SD & Mean & SD & \\
\hline Age & & 32.73 & 5.65 & 31.80 & 6.30 & - 0.673 \\
\hline \multirow{2}{*}{\multicolumn{2}{|c|}{$\begin{array}{l}\text { Disease duration (years) } \\
\text { Median (IQR) }\end{array}$}} & $6(3-8)$ & & $5(3-7)$ & & • 0.545 \\
\hline & & $\mathbf{N}$ & $\%$ & $\mathbf{N}$ & $\%$ & \\
\hline \multirow{3}{*}{ Sex } & Male & 4 & $26.7 \%$ & 6 & $40 \%$ & \multirow{2}{*}{ II 0.439} \\
\hline & Female & 11 & $73.3 \%$ & 9 & $60 \%$ & \\
\hline & & Mean & SD & Mean & SD & \\
\hline \multicolumn{2}{|l|}{ Wide spread pain index (WPI) } & 16.27 & 1.62 & 14.87 & 2.23 & - 0.673 \\
\hline \multicolumn{2}{|l|}{ Symptom severity scale (SSS) } & 10.75 & 1.75 & 10.03 & 1.45 & $\cdot 1.000$ \\
\hline \multicolumn{2}{|l|}{ Visual analogue scale (VAS) } & 7.73 & 1.28 & 7.00 & 0.93 & - 0.059 \\
\hline \multicolumn{2}{|l|}{ Tender point score } & 16.27 & 1.62 & 14.87 & 2.23 & • 0.059 \\
\hline \multicolumn{2}{|l|}{$\begin{array}{l}\text { Revised fibromyalgia impact } \\
\text { questionnaire (FIQR) }\end{array}$} & 55.92 & 3.50 & 55.66 & 3.52 & • 0.841 \\
\hline \multicolumn{2}{|l|}{ (HADS) Depression sub-score } & 7.13 & 2.73 & 5.93 & 1.77 & - 0.078 \\
\hline \multicolumn{2}{|l|}{ (HADS) Anxiety sub-score } & 12.6 & 1.99 & 10.33 & 1.95 & - 0.737 \\
\hline
\end{tabular}

Statistical methods used: - Independent Student t- test, IIChi square test. HADS: Hospital Anxiety and Depression Scale.

Post-intervention in the rTMS therapy group, the VAS, Tender point score and FIQR were highly significantly lower than corresponding values before treatment. While, HADS anxiety and depression subscales demonstrated a significant post-intervention reduction (Table 2).

Table (2): Comparison of clinical assessment scales before and after treatment in r-TMS therapy group

\begin{tabular}{|l|c|c|c|}
\hline \multicolumn{1}{|c|}{ Clinical scores } & $\begin{array}{c}\text { r-TMS therapy } \\
\text { group (pre- } \\
\text { treatment) } \\
(\mathbf{n}=\mathbf{1 5}) \\
(\mathbf{m e a n} \pm \mathbf{S D})\end{array}$ & $\begin{array}{c}\text { r-TMS therapy } \\
\text { group (post- } \\
\text { treatment) } \\
(\mathbf{n = 1 5 )} \\
(\mathbf{m e a n} \pm \mathbf{S D})\end{array}$ & p -value \\
\hline Visual analogue scale (VAS) & $7.73 \pm 1.28$ & $3.33 \pm 1.11$ & $\cdot<0.001^{* *}$ \\
\hline Tender point score & $16.27 \pm 1.62$ & $11.40 \pm 2.06$ & $\cdot<0.001^{* *}$ \\
\hline $\begin{array}{l}\text { Revised fibromyalgia impact } \\
\text { questionnaire (FIQR) }\end{array}$ & $55.92 \pm 3.50$ & $43.03 \pm 2.79$ & $\cdot<0.001^{* *}$ \\
\hline (HADS) Depression sub-score & $7.13 \pm 2.73$ & $4.33 \pm 1.76$ & $\cdot 0.044^{*}$ \\
\hline (HADS) Anxiety sub-score & $12.6 \pm 1.99$ & $6.13 \pm 1.30$ & $\cdot 0.048^{*}$ \\
\hline
\end{tabular}

Statistical methods used: • paired Student t test. *: Significant, **: Highly significant, HADS: Hospital Anxiety and Depression Scale.

In the tDCS therapy group the VAS, Tender point score and FIQR were highly significantly lower than corresponding values before treatment. While, HADS anxiety and depression subscales demonstrated a significant post-intervention reduction (Table 3). 
Table (3): Comparison of clinical assessment scales before and after treatment in t-DCS therapy group

\begin{tabular}{|l|c|c|c|}
\hline \multicolumn{1}{|c|}{ Clinical scores } & $\begin{array}{c}\text { t-DCS therapy group } \\
(\text { pre-treatment) } \\
(\mathbf{n}=\mathbf{1 5}) \\
(\text { mean } \pm \text { SD) }\end{array}$ & $\begin{array}{c}\text { t-DCS therapy group (post- } \\
\text { treatment) } \\
(\mathbf{n}=\mathbf{1 5}) \\
(\text { mean } \pm \text { SD) }\end{array}$ & p -value \\
\hline Visual analogue scale (VAS) & $7.00 \pm 0.93$ & $3.93 \pm 1.03$ & $\cdot<0.001^{* *}$ \\
\hline Tender point score & $14.87 \pm 2.23$ & $11.00 \pm 1.69$ & $\cdot<0.001^{* *}$ \\
\hline $\begin{array}{l}\text { Revised fibromyalgia impact } \\
\text { questionnaire (FIQR) }\end{array}$ & $55.66 \pm 3.52$ & $45.59 \pm 3.25$ & $\cdot<0.001^{* *}$ \\
\hline $\begin{array}{l}\text { (HADS) Depression sub- } \\
\text { score }\end{array}$ & $5.93 \pm 1.77$ & $3.93 \pm 1.09$ & $\cdot 0.050^{*}$ \\
\hline (HADS) Anxiety sub-score & $10.33 \pm 1.95$ & $5.07 \pm 1.87$ & $\cdot 0.016^{*}$ \\
\hline
\end{tabular}

Statistical methods used: • Paired Student t test. *: Significant, **: Highly significant, HADS: Hospital Anxiety and Depression Scale.

On comparing post-intervention clinical outcome measures, all scores were found to be statistically comparable in both groups except for the FIQR score which showed a significant statistical difference (Table 4).

Table (4): Comparison of VAS, Tender point score, FIQR and HADS after treatment in both groups

\begin{tabular}{|c|c|c|c|}
\hline Clinical scores & $\begin{array}{c}\text { r-TMS therapy group } \\
\text { (post treatment) } \\
(\mathbf{n}=15)\end{array}$ & $\begin{array}{c}\text { t-DCS therapy group } \\
\text { (post treatment) } \\
(\mathrm{n}=15)\end{array}$ & p -value \\
\hline Visual analogue scale (VAS) & $3.33 \pm 1.11$ & $3.93 \pm 1.03$ & - 0.137 \\
\hline Tender point score & $11.40 \pm 2.06$ & $11.00 \pm 1.69$ & $\cdot 0.566$ \\
\hline $\begin{array}{l}\text { Revised fibromyalgia impact } \\
\text { questionnaire (FIQR) }\end{array}$ & $43.03 \pm 2.79$ & $45.59 \pm 3.25$ & - $0.028^{*}$ \\
\hline (HADS) Depression sub-score & $4.33 \pm 1.76$ & $3.93 \pm 1.09$ & - 0.315 \\
\hline (HADS) Anxiety sub-score & $6.13 \pm 1.30$ & $5.07 \pm 1.87$ & - 0.900 \\
\hline
\end{tabular}

Statistical methods used: — Independent Student t- test. *: Significant, HADS: Hospital Anxiety and Depression Scale.

As regards the comparison of the degree of change in all assessed scores post-treatment between both groups, a high statistically significant difference was found between both groups in the VAS, Tender point score and FIQR. HADS anxiety and depression subscales demonstrated a significant difference (Table 5).

Table (5): Comparison of change of VAS, Tender point score, FIQR and HADS post-treatment in both groups

\begin{tabular}{|l|c|c|c|}
\hline \multicolumn{1}{|c|}{ Clinical scores } & $\begin{array}{c}\text { r-TMS therapy } \\
\text { group (change } \\
\text { post- treatment) } \\
(\mathbf{n = 1 5 )} \\
\text { Median (IQR) }\end{array}$ & $\begin{array}{c}\text { t-DCS therapy group } \\
\text { (change post- treatment) } \\
(\mathbf{n = 1 5}) \\
\text { Median (IQR) }\end{array}$ & P-value \\
\hline Visual analogue scale (VAS) & $-4(-5--4)$ & $-3(-4--3)$ & $\ddagger<0.001^{* *}$ \\
\hline Tender point score & $-5(-6--4)$ & $-4(-5--3)$ & $\ddagger<0.001^{* *}$ \\
\hline $\begin{array}{l}\text { Revised fibromyalgia impact } \\
\text { questionnaire (FIQR) }\end{array}$ & $-12.3(-14.4--11.6)$ & $-10(-11.4--9)$ & $\ddagger<0.001^{* *}$ \\
\hline $\begin{array}{l}\text { (HADS) Depression sub- } \\
\text { score }\end{array}$ & $-3.80 \pm 1.86$ & $-2.00 \pm 1.00$ & $-0.030^{*}$ \\
\hline (HADS) Anxiety sub- score & $-6.47 \pm 1.64$ & $-4.87 \pm 1.51$ & $-0.021^{*}$ \\
\hline
\end{tabular}

Statistical methods used: \$ Mann Whitney test, - Independent Student t- test. *: Significant, **: Highly significant, HADS: Hospital Anxiety and Depression Scale. 


\section{DISCUSSION}

The current study demonstrated that 8 sessions of high-frequency rTMS (2/week for 4 consecutive weeks) and 8 daily sessions of anodal tDCS in 1ry FM patients resulted in a marked improvement of pain VAS, functional abilities, and different aspects of quality of life assessed by FIQ, anxiety and depression subscales of HADS in addition to tender point score at the end of the treatment schedule for both techniques. However, the group of rTMS therapy showed a higher degree of change of all clinical assessment scores post-treatment than the group of tDCS.

The results of this study demonstrated that the use of neuromodulatory techniques in the management of 1ry FM can improve pain, functionally related disabilities, and psychological status of patients, especially for rTMS protocol. The addition of rTMS and tDCS to the management protocol of FM patients can improve the quality of the life and reduce the side effects of drugs.

Our results in the rTMS therapy group are comparable to those of Altas et al. ${ }^{\text {(26) }}$, in which 30 patients with FM were recruited and underwent 15 sessions of 10-Hz rTMS to the left M1 (10 patients), left-hemisphere dorsolateral prefrontal cortex (DLPFC) (10 patients), and sham (10 patients). Significant improvements was reported in pain, quality of life, and depression scores in all three groups. However, the decrease in VAS scores was significantly prominent in patients who received rTMS over left M1, whereas improvement in physical function was more significant in patients who received rTMS over DLPFC. Besides, the results of the rTMS group matched with those of Mhalla et al. ${ }^{(12)}$, who assessed the long-term maintenance of analgesic effect of high-frequency rTMS applied lo left M1 in thirty patients with FM (sixteen in the active stimulation group compared to fourteen in the sham stimulation group). The active rTMS group reported a significant reduction in pain intensity from day 5 to week 25 . In addition to a long-term improvement of morning tiredness, fatigue, walking, and sleep. Similarly, in the study of Passard et al. (27), thirty patients with FM were randomly divided into two groups (15 received active rTMS and 15 received sham stimulation, applied to the left M1 over 10 daily sessions). Active rTMS group showed significantly reduced pain starting from the fifth session besides improved several aspects of quality of life lasting for up to 2 weeks post-intervention. The study of Fitzgibbon et al. ${ }^{(28)}$, provided evidence that 4-weeks daily sessions (5/week) of high frequency (10HZ) rTMS (total of 20 sessions) applied to the left DLPFC in 26 patients with FM were effective in improving fatigue and pain intensity.
Our study results in the tDCS group matched those of Fregni et al. ${ }^{(29)}$, who found that five sessions of anodal tDCS over left M1 were effective as compared to the placebo group in reducing pain and improving quality of life in patients with FM and persisted for 3 weeks post-intervention. Our results also agree with Jales Junior et al. ${ }^{\left({ }^{(3)}\right)}$ who studied the effects of the application of $1 \mathrm{~mA}$ of anodal stimulation of tDCS over left M1 area, given once a week in a total of 10 consecutive weeks to a group of 10 females with FM. The sham group included 10 patients with FM that received no electric pulses. They reported a significant decrease in the FIQ and the VAS scores. In addition to a significant reduction in trigger points' measurement and functional capacity, physical and emotional aspects of the short-form survey (SF36) domains in the effective group. Valle et al. ${ }^{(9)}$, compared the efficacy of M1 and DLPFC tDCS stimulation applied to forty-one females with chronic FM refractory to medical treatment. Both groups displayed improvements in VAS pain score and quality of life assessed by FIQ at the end of the treatment protocol. Even though, only M1 stimulation resulted in long-lasting clinical benefits at 30 and 60 day's post-intervention.

A systematic review study that determines the effects of tDCS and TMS on the main symptoms of patients with FM concluded that the application of tDCS to the motor cortex can decrease pain in the short and medium-term in patients with FM. Both techniques showed improvements in pressure pain threshold, and quality of life when applied to the motor cortex, and in fatigue scales when applied to the DLPFC. While the effects of both modalities on anxiety and depression were undetermined ${ }^{(31)}$.

The primary motor cortex has been the most studied target to improve pain $(\mathbf{1 1 , 2 6 , 2 7 , 2 9 , 3 0 )}$. The reduction in both pain and depression/anxiety has been observed by targeting the left M1 area in our patients via either high-frequency rTMS or tDCS. This may be explained by that depression and anxiety are consequences of chronic pain and that the decrease of pain by treatment via neuromodulatory techniques may improve the psychological status of patients. The brain regions involved in pain and depression are closely related. The study of Vishne et al. ${ }^{(32)}$, reported a bidirectional relationship between pain and depression. The intensity of the nociceptive pain stimulus has been associated with central sensitization activation and depression. Thus, the antidepressant effect of both neuromodulatory techniques observed in this study may be a sequential effect of improving pain, and not the depressive symptom itself. So, it can be concluded that M1 cortex stimulation could be a target for the treatment of anxiety and depression in patients with FM. 
The mechanism of pain relief through cortical stimulation by rTMS is based on the modification of neuronal excitability. rTMS induces alterations in the activity of cortical and subcortical brain structures that are contributing to pain processing and modulation, including the periaqueductal gray matter, orbitofrontal cortices, anterior cingulate and, medial thalamus ${ }^{(33)}$.

Positive effects in tDCS in FM patients may be attributed to its neurophysiological effects including facilitation of synaptic efficacy, expression of neurotrophic factors, and improvements in regional cerebral blood flow ${ }^{(34)}$. The improvement in pain modulation resulting from tDCS is attributed to the long term potentiation (LTP) of new adaptive synaptic connection ${ }^{(35)}$.

There were some limitations to this study. First, the small sample size that could give a chance of type I error. Second, the participants were aware of their treatments, which could give some expectations of treatment benefit and thus influence the assessment of our results due to a lack of blinding and a control group. Third, we studied only primary FM patients. So, we could not represent the characteristics of all FM patients and these results may not be generalizable. Another important limitation is that we could not determine the optimal number of stimulation sessions, treatment interval, and booster regimen.

\section{CONCLUSION}

The use of neuromodulatory techniques including rTMS and tDCS targeting the left primary motor cortex are potentially effective alternative treatment options in fibromyalgia patients. Both techniques were effective in producing marked improvement in symptoms of pain, functional abilities, and psychological status in patients with 1ry FM. A major advantage of both techniques over pharmacological treatment would be their excellent tolerability and fewer side effects. However, further studies are required to standardize the optimal parameters and protocols of stimulation. In particular, the effects of right versus left stimulation should be assessed to investigate the lateralization of the effects. Future studies should also confirm the long term sustained analgesic effects.

\section{REFERENCES}

1. Bennett $\mathbf{R}$ (2005): Fibromyalgia: present to future. Curr Rheumatol Rep., 7(5): 371-376.

2. Bannwarth B, Blotman F, Roué-Le Lay $\mathrm{K}$ et al. (2009): Fibromyalgia syndrome in the general population of France: a prevalence study. Joint Bone Spine, 76 (2): 184-187.

3. Weir P, Harlan G, Nkoy F et al. (2006): The incidence of fibromyalgia and its associated comorbidities: a population-based retrospective cohort study based on International Classification of Diseases, 9th Revision codes. J Clin Rheumatol., 12(3): 124-128.
4. Mease P, Clauw D, Arnold $L$ et al. (2005): Fibromyalgia syndrome. J Rheumatol., 32 (11): 22702277.

5. Lautenbacher S, Rollman G (1997): Possible deficiencies of pain modulation in fibromyalgia. Clin $\mathrm{J}$ Pain, 13 (3): 189-196.

6. Staud R, Cannon R, Mauderli A et al. (2003): Temporal summation of pain from mechanical stimulation of muscle tissue in normal controls and subjects with fibromyalgia syndrome. Pain, 102(1-2): 8795.

7. Staud R, Robinson M, Vierck $\mathbf{J}$ et al. (2003): Diffuse noxious inhibitory controls (DNIC) attenuate temporal summation of second pain in normal males but not in normal females or fibromyalgia patients. Pain, 101: 167174.

8. Mhalla A, de Andrade D, Baudic S et al. (2010): Alteration of cortical excitability in patients with fibromyalgia. Pain, 149 (3): 495-500.

9. Valle A, Roizenblatt S, Botte S et al. (2009): Efficacy of anodal transcranial direct current stimulation (tDCS) for the treatment of fibromyalgia: results of a randomized, sham-controlled longitudinal clinical trial. J Pain Manag., 2: 353-361.

10. Antal A, Terney D, Kuhnl S et al. (2010): Anodal transcranial direct current stimulation of the motor cortex ameliorates chronic pain and reduces short intracortical inhibition. J Pain Symptom Manage, 39 (5): 890-903.

11. Short E, Borckardt J, Anderson B et al. (2011): Ten sessions of adjunctive left prefrontal rTMS significantly reduces fibromyalgia pain: a randomized, controlled pilot study. Pain, 152 (11): 2477-2484.

12. Mhalla A, Baudic S, de Andrade D et al. (2011): Longterm maintenance of the analgesic effects of transcranial magnetic stimulation in fibromyalgia. Pain, 152 (7): 1478-1485.

13. Marlow N, Bonilha H, Short E (2013): Efficacy of transcranial direct current stimulation and repetitive transcranial magnetic stimulation for treating fibromyalgia syndrome: a systematic review. Pain Pract., 13(2): 131-145.

14. Barker A, Jalinous R, Freeston I (1985): Non-invasive magnetic stimulation of human motor cortex. Lancet, 325(8437):1106-1107.

15. Jodoin M, Rouleau D, Larson-Dupuis C et al. (2017): The clinical utility of repetitive transcranial magnetic stimulation in reducing the risks of transitioning from acute to chronic pain in traumatically injured patients. Prog Neuro psycho pharmacol Biol Psychiatry, 87:322331.

16. Nitsche M, Paulus W (2000): Excitability changes induced in the human motor cortex by weak transcranial direct current stimulation. J Physiol., 527(3):633-639.

17. Thomas A, Graham K, Prato F et al. (2007): A randomized, double-blind, placebo-controlled clinical trial using a low frequency magnetic field in the treatment of musculoskeletal chronic pain. Pain Res Manag., 12: 249-258.

18. Maestú C, Blanco M, Nevado A et al. (2013): Reduction of pain thresholds in fibromyalgia after very low-intensity magnetic stimulation: a double-blinded, randomized placebo-controlled clinical trial. Pain Res Manag., 18: 101-106. 
19. Villamar M, Wivatvongvana $P$, Patumanond $\mathrm{J}$ et al. (2013): Focal modulation of the primary motor cortex in fibromyalgia using $4 \times 1$-ring high-definition transcranial direct current stimulation (HD-tDCS): immediate and delayed analgesic effects of cathodal and anodal stimulation. J Pain, 14(4):371-383.

20. Wolfe F, Clauw D, Fitzcharles $M$ et al. (2010): The American College of Rheumatology preliminary diagnostic criteria for fibromyalgia and measurement of symptom severity. Arthritis Care Res., 62 (5):600-610.

21. Smit D, Posthuma D, Boomsma D et al. (2005): Heritability of background EEG across the power spectrum. Psychophysiology, 42 (6): 691- 697.

22. Hawker G, Mian S, Kendzerska T et al. (2012): Measures of adult pain: Visual Analog Scale for Pain (VAS Pain), numeric rating scale for pain (nrs pain), mcgill pain questionnaire (mpq), short-form mcgill pain questionnaire (sf-mpq), chronic pain grade scale (cpgs), short form-36 bodily pain scale (sf-36 bps), and measure of intermittent and constant osteoarthritis pain (icoap). Arthrit Care Res., 63(11):240-252.

23. Bennett R, Friend R, Jones $K$ et al. (2009): The Revised Fibromyalgia Impact Questionnaire (FIQR): validation and psychometric properties. Arthritis Res Ther., 11 (4):120-126.

24. Lundberg G, Gerdle B (2002): Tender point scores and their relations to signs of mobility, symptoms, and disability in female home care personnel and the prevalence of fibromyalgia syndrome. J Rheumatol., 29 (3): 603-613.

25. Zigmond A, Snaith R (1983): The hospital anxiety and depression scale. Acta Psychiat Scand., 67 (6): 361-370.

26. Altas E, Askin A, Besiroglu L et al. (2019): Is highfrequency repetitive transcranial magnetic stimulation of the left primary motor cortex superior to the stimulation of the left dorsolateral prefrontal cortex in fibromyalgia syndrome? Somatosens Mot Res., 36 (1):56-62.

27. Passard A, Attal N, Benadhira R et al. (2007): Effects of unilateral repetitive transcranial magnetic stimulation of the motor cortex on chronic widespread pain in fibromyalgia. Brain, 130 (10): 2661-2670.

28. Fitzgibbon B, Hoy K, Knox L et al. (2018): Evidence for the improvement of fatigue in fibromyalgia: A 4-week left dorsolateral prefrontal cortex repetitive transcranial magnetic stimulation randomized-controlled trial. Eur J Pain, 22 (7): 1255-1267.

29. Fregni F, Gimenes R, Valle A et al. (2006): A randomized, sham-controlled, proof of principle study of transcranial direct current stimulation for the treatment of pain in fibromyalgia. Arthritis Rheum: Official Journal of the American College of Rheumatology, 54(12): 39883998.

30. Jales Junior L, Costa M, Jales Neto L et al. (2015): Transcranial direct current stimulation in fibromyalgia: effects on pain and quality of life evaluated clinically and by brain perfusion scintigraphy. Revista Dor., 16(1):3742.

31. Conde-Antón Á, Hernando-Garijo I, Jiménez-delBarrio S et al. (2020): Efectos de la estimulación transcraneal por corriente directa y de la estimulación magnética transcraneal en pacientes con fibromialgia. Revisión sistemática. Neurología, https://doi.org/10.1016/j.nrl.2020.07.024

32. Vishne T, Fostick L, Silberman A et al. (2008): Fibromyalgia among major depression disorder females compared to males. Rheumatol Int., 28(9): 831-836.

33. Lefaucheur $\mathbf{J}$ (2008): Use of repetitive transcranial magnetic stimulation in pain relief. Exp Rev Neurother., 8(5):799-808.

34. Brunoni A, Nitsche $M$, Bolognini $N$ et al. (2012): Clinical research with transcranial direct current stimulation (tDCS): challenges and future directions. Brain stimul., 5(3): 175-195.

35. Fregni F, Freedman S, Pascual-Leone A (2007): Recent advances in the treatment of chronic pain with non-invasive brain stimulation techniques. Lancet Neurol., 6 (2): 188-91. 